\title{
Structural Interpretation of Metastable States in MbNO
}

\author{
Maksym Soloviov, Akshaya K. Das and Markus Meuwly* \\ Department of Chemistry, University of Basel, \\ Klingelbergstrasse 80, 4056 Basel, Switzerland \\ E-mail: m.meuwly@unibas.ch
}

\begin{abstract}
Nitric oxide binding and unbinding from myoglobin $(\mathrm{Mb})$ is central to the function of the protein. Using reactive molecular dynamics (MD) simulations the dynamics following NO dissociation is characterized both, in time and in space. The time scales for ligand rebinding include two processes on the 10 ps and 100 ps time scale which agrees with recent optical and X-ray absorption experiments. Explicitly including the iron-out-of-plane (Fe-oop) coordinate is essential for meaningful interpretation of the data. Also, the proposed existence of an "Fe-oop/NO-bound" state is confirmed and assigned to $\mathrm{NO}$ at a distance of $\approx 3 \AA$ away from the iron atom. However, calculated XANES spectra suggest that it is difficult to distinguish between NO close but chemically unbound to the heme-Fe and positions further away in the primary site. Another elusive state, Fe-ON coordination, is not observed experimentally because it is masked by the energetically more favorable but dissociative ${ }^{4} A$ state in this region which makes the Fe-ON local minimum unobservable in wild type Mb. However, suitable active site mutations may stabilize this state.
\end{abstract}

${ }^{*}$ To whom correspondence should be addressed 
Following the motion of photodissociated ligands in globular proteins has a long history. Small molecules which can reversibly bind to the protein active center are ideal and sensitive probes of the interior of such complex systems. Nitric oxide (NO) is a physiologically relevant ligand, ${ }^{8-10}$ involved in modulating blood flow, thrombosis, and neural activity. Experimentally, the binding kinetics of NO to the heme-group in myoglobin (Mb) has been studied extensively with time resolved spectroscopies, ranging from UV/visible to the mid-IR ${ }^{11-17}$ and resonance Raman ${ }^{14}$ techniques. In all cases the rebinding kinetics is multi-exponential with time constants ranging from sub-picoseconds to several hundred picoseconds. However, they can be grouped into two broad classes: processes on the 10 ps time scale and those on the 100 ps time scale. ${ }^{12,14-16,18,19}$

Recent experiments have followed the interplay between the Fe-out-of-plane (Fe-oop) and the NO-ligand motion in a time resolved fashion. ${ }^{5,14,16}$ This work points towards a direct coupling between these two degrees of freedom on the 10 to 100 ps time scale. What re- 
mains unclear is the structural characterization of this state. Furthermore, earlier studies have attempted to characterize the metastable Fe-ON state ${ }^{20}$ and the question remains why this commonly observed motif in model compounds ${ }^{21}$ is absent in the protein environment despite a stabilization of $5 \mathrm{kcal} / \mathrm{mol}$ or more. ${ }^{22}$

The atomistic dynamics is essential for protein function and provides the basis to interpret the time scales involved at a structural level. Molecular dynamics (MD) simulations are ideal for addressing such questions. Together with validated energy functions such simulations can provide the "missing link" between time resolved experiments and the underlying atomic motions. ${ }^{4,23,24}$ For NO interacting with heme in Mb such potential energy surfaces (PESs) for the bound ${ }^{2} A$ and ligand-unbound ${ }^{4} A$ state are available. ${ }^{25}$

Running a statistically significant number of QM/MM trajectories from which to analyze and resolve the interplay of the motions involved is beyond current computational methods for systems such as MbNO. For quantitative and meaningful computational investigations, suitable representations that preserve the accuracy of the interpolated PES are used in the simulations. Here, a parameter-free, reproducing kernel Hilbert space (RKHS)-based representation which exactly reproduces the reference data from quantum chemical calculations is employed (for details on the PESs, see the SI) ${ }^{26,27}$ RKHS is based on smoothness criteria of the interpolant and has been successfully applied to study van der Waals complexes. ${ }^{28,29}$

The present work investigates the reactive dynamics between the ${ }^{4} A$ and ${ }^{2} A$ PESs following photodissociation of the NO ligand from the heme-iron in Mb. Due to the close energetic ordering of the two states and the existence of two substates on the ${ }^{2} A$ PES, interesting dynamics can be expected. From comparison with experimentally determined rebinding time scales and the spectroscopies, structural questions underlying the molecular dynamics can be addressed. 


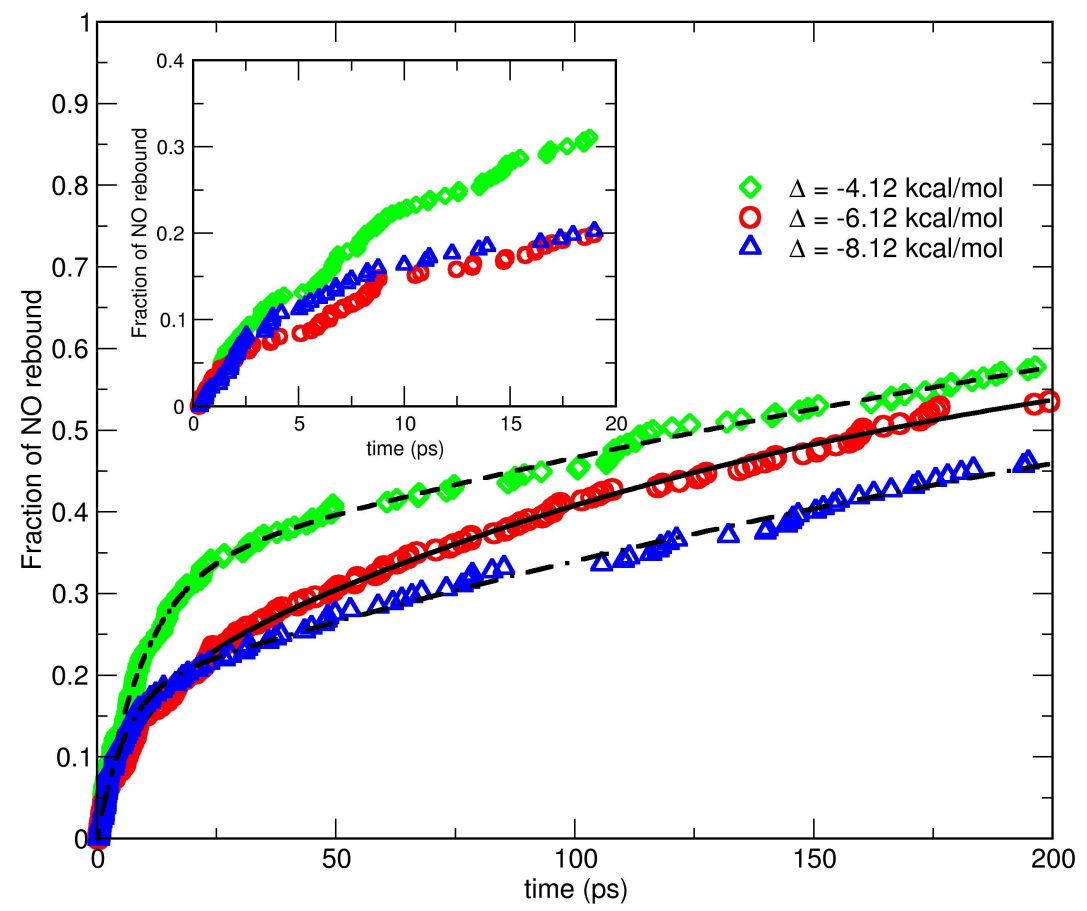

Figure 1: The kinetics and the corresponding exponential fits for NO rebinding to the hemeFe after photoexcitation. Simulations were run for three different values of the asymptotic shift $\Delta$.

For following the reactive dynamics between the ${ }^{2} A$ and ${ }^{4} A$ states, 300 independent simulations were run for a maximum time of 200 ps or until the bound Fe-NO state was formed. Photodissociation was induced by instantaneously switching the force field to the ${ }^{4} A$ state ${ }^{30}$ which introduces around $50 \mathrm{kcal} / \mathrm{mol}$ of energy, ${ }^{25}$ comparable with the photon energies used in experiments (49 to $81 \mathrm{kcal} / \mathrm{mol}$ ). ${ }^{31}$ The rebinding kinetics is reported in Figure 1 and shows the fraction of rebound $\mathrm{NO}$ as a function of time. The rebinding kinetics on the sub-nanosecond time scale follows a multiexponential decay with two time constants $\tau_{1} \approx 10$ ps and $\tau_{2} \approx 150 \mathrm{ps}$, also depending on the asymptotic separation $\Delta$ of the two states (see Table I SI). The shift $\Delta$ relates the asymptotic energies of the ${ }^{2} A$ and the ${ }^{4} A$ PESs for NO at infinite separation from the heme-Fe (see SI).

Typical ligand trajectories are shown in Figure 2. Panel A reports rebinding on the 1 ps time 
scale whereas panels $\mathrm{B}$ and $\mathrm{C}$ are representative of rebinding time scales $\tau_{1}$ and $\tau_{2}$. For $\tau_{1}$ the ligand only samples the immediate neighborhood of the heme-iron before rebinding. On the 100 ps time scale rebinding occurs from regions further away. For $\Delta=-6.1 \mathrm{kcal} / \mathrm{mol}$ the total number of rebound trajectories is 17 (rebinding within 2 ps), $28(2 \leq t<10$ ), $115(10 \leq t<200)$, and 140 rebind on time scales longer than 200 ps, i.e. the rebinding efficiency within $200 \mathrm{ps}$ is $\sim 55 \%$. This compares with a rebound fraction of $75 \%$ on the 200 ps time scale from recent XAS measurements. ${ }^{5}$

The simulations described so far used the PESs fitted to the DFT-data and including environmental effects (see SI). Corresponding simulations with 200 independent runs on the DFT-only PESs yield rebinding of 195 trajectories within 200 ps with rebinding times of 2 and 15 ps, i.e. one order of magnitude more rapid than with the refined PES treating the Fe-oop coordinate more accurately and explicitly. This finding is also consistent with previous simulations based on DFT-only PESs. ${ }^{19,32}$ Hence, explicitly including the Fe-oop motion is essential for quantitative results.
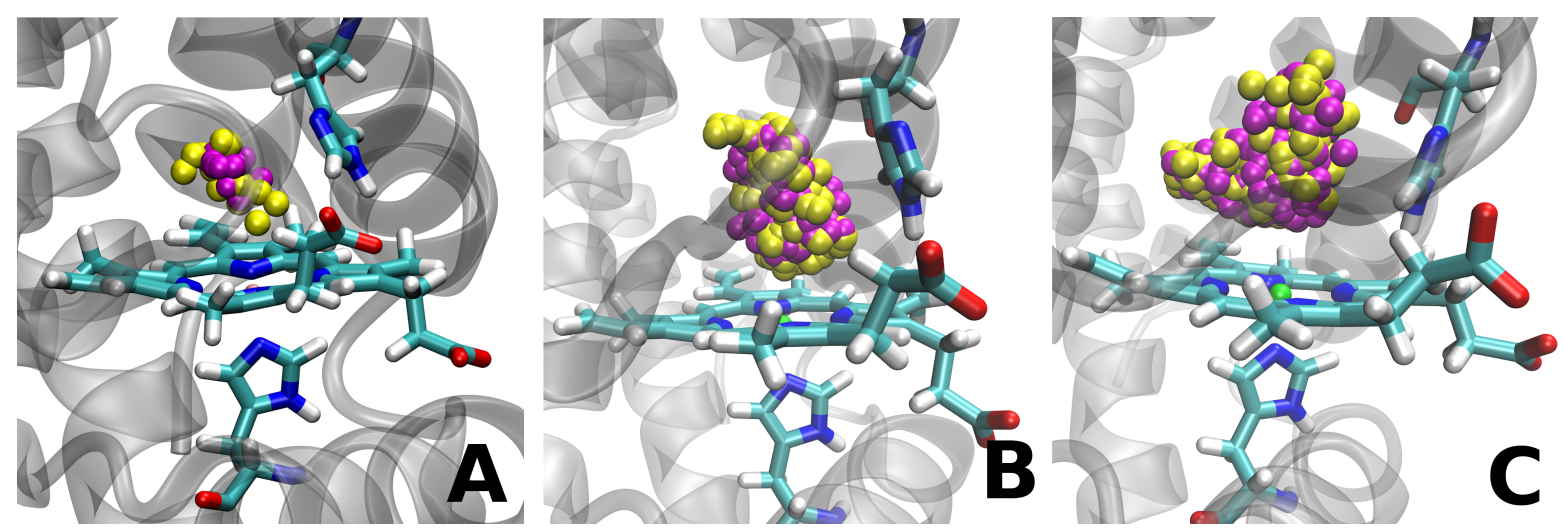

Figure 2: Typical trajectories for different rebinding time scales. NO positions are shown in yellow $(\mathrm{N})$ and magenta $(\mathrm{O})$. (A) - the picosecond process $(\tau=1.6 \mathrm{ps}),(\mathbf{B})$ - the 10 picosecond process $(\tau=42.1 \mathrm{ps}),(\mathbf{C})$ - the 100 picosecond process $(\tau=160.2 \mathrm{ps})$.

A complementary view of the different rebinding time scales can be gained from analyzing the maximal distance between the Fe and the ligand sampled during a rebinding simulation. 

within 10 ps and longer than 10 ps. The individually normalized probability distribution functions (pdfs) are shown in Figure S5 (see SI). No NO migration to neighboring xenonbinding sites is found on the 200 ps time scale because, e.g., escape to the closest Xe-site (Xe4) occurs within 1 to $10 \mathrm{~ns} .^{33,34}$

Previous experimental and computational investigations ${ }^{12,19,30,32,35-37}$ found that the recombination kinetics of MbNO is non-exponential, involving two to three time scales, depending on the model used. The earliest works report time scales of 27.6 ps and 279.3 ps in a double exponential fit. ${ }^{12}$ Later, optical and infrared experiments found short time scales ranging from 5 to 30 ps and longer time scales between 100 and 200 ps. ${ }^{35-37}$ The experiments all agree on the existence of two sub-nanosecond time scales which differ by about one order of magnitude. Previous computations with reactive force fields find typically somewhat shorter rebinding times, between 5 ps and $20 \mathrm{ps},{ }^{19,32}$ depending on the asymptotic separation $\Delta$ used. $^{38}$ The current simulations find multiple time scales in the 10 to 100 ps range in agreement with optical and infrared experiments. Also, a more rapid component on the 1 to 2 ps time scale is present. The influence of Fe-doming is clearly visible in the rebinding kinetics, see Figure 1. This validates the force fields used here and allows to address a number of hypotheses put forward recently.

The Fe-oop, NO-bound state: First, direct contact with a recently proposed transient structure in the ${ }^{2} A$ state can be made. Picosecond time resolved ${ }^{14}$ and $\mathrm{XAS}^{5}$ experiments have suggested that the Fe-out-of-plane (Fe-oop) and the NO-ligand motion are closely coupled. The interpretation of the experiments provided evidence for an Fe-oop, ligand bound structure with a lifetime of $30 \pm 10$ ps. The present simulations on the ${ }^{2} A$ PES alone indeed show transient stabilization of such a state. The maximal lifetime found was 27 ps and $41.7 \%$ of the trajectories showed such a state (see Figure S3). Despite underestimating the 

of such a state.

The Fe-ON state: Another structurally elusive state which has been found for heme-model compounds is the Fe-ON isomer. ${ }^{21,39,40}$ Such a structure is sufficiently stabilized (by several kcal/mol) in NO-bound $\mathrm{Mb}$ which should make it observable in infrared experiments. ${ }^{22}$ However, no such state was found in experiments. ${ }^{20}$ Simulations on the ${ }^{2} A$ state alone show that the ligand is stabilized for tens of picoseconds in the Fe-ON state. However, when using the reactive ${ }^{2} A+{ }^{4} A$ PES with a shift of $\Delta=-6.1 \mathrm{kcal} / \mathrm{mol}$ (which best reproduces the DFT energies) the Fe-ON configuration can not be stabilized because in the region of the $\mathrm{Fe}-\mathrm{ON}$ minimum the repulsive ${ }^{4} A$ state is lower in energy than the ${ }^{2} A$ state. Hence, the ${ }^{2} A$ minimum of the Fe-ON state is masked by the ${ }^{4} A$ state and can not be stabilized in wt Mb.

The asymptotic separation $\Delta$ depends on the chemical environment around the heme-group. Hence, $\Delta$ changes if the environment is modified, e.g. through mutation of amino acids or embedding the heme-group into a different protein. The effect of this can be quantified with simulations on the reactive ${ }^{2} A+{ }^{4} A$ PES with different values for $\Delta$. Reducing the asymptotic separation by $5 \mathrm{kcal} / \mathrm{mol}(\Delta=-11.1 \mathrm{kcal} / \mathrm{mol})$ further destabilizes the $\mathrm{Fe}-\mathrm{ON}$ state (Figure SI 6A). For $\Delta=-6.1 \mathrm{kcal} / \mathrm{mol}$ (Figure SI 6B) the Fe-ON state is insignificantly sampled and the system rebinds efficiently into the Fe-NO state. Contrary to that, reducing the asymptotic separation to $\Delta=-1.1 \mathrm{kcal} / \mathrm{mol}$ (Figure SI $6 \mathrm{C}$ ) the Fe-ON state is populated for extended periods during which spectroscopic characterization of this state should be possible. As an illustration of this, recent spectroscopic work found a 2.5 times slower dynamics of $\mathrm{NO}$ rebinding in Mb compared to cytochrome c due to different active site architecture. ${ }^{41}$ Based on transition state theory, a factor of 2.5 corresponds to $\approx 0.5$ $\mathrm{kcal} / \mathrm{mol}$ in energy. The thermodynamic stability of Mb upon mutation has been found to change by -2 to $+6 \mathrm{kcal} / \mathrm{mol}^{42}$ which suggests that modifications in the active site can 
potentially stabilize the $\mathrm{Fe}-\mathrm{ON}$ conformation through differential stabilization of the bound state relative to the unbound state.

Structural interpretation of rebinding time scales: With the coordinates from the reactive MD simulations it is possible to provide a structural interpretation of the $\approx 10$ ps and $\approx 100$ ps time scale found in the rebinding simulations. It is of particular interest to investigate in what respect(s) the dynamics between these two states differ. Probability distribution functions of the a) NO-ligand, b) all active-site residues, and c) all His64 side chain atoms projected onto the heme plane are shown in Figure 3.

The pdfs show that the NO-ligand explores a much larger space for long rebinding times (Figure 3 upper right hand panel) than for short rebinding times (Figure 3 upper left hand panel). Furthermore, the NE2 atom of residue His64 occupies two clearly distinguishable states $(\mathrm{A}$ and $\mathrm{B})$ in trajectories with long rebinding times which are absent for rebinding on $\tau_{1}=10$ ps (Figure 3 middle row). The middle row emphasizes that for time scales $\tau_{1}$ the His64 NE2 atom occupies space away from the iron (located at $(0,0)$ ) whereas for longer time scales the atom pushes in towards the heme-Fe by almost $2 \AA$ which hinders ligand rebinding. The estimated forward $\Delta G_{\mathrm{A} \rightarrow \mathrm{B}}^{\mathrm{NE} 2}=2.5 \mathrm{kcal} / \mathrm{mol}$ and reverse $\Delta G_{\mathrm{B} \rightarrow \mathrm{A}}^{\mathrm{NE} 2}=4.0 \mathrm{kcal} / \mathrm{mol}$ barriers suggest that on the 100 ps time scale state A is destabilized relative to state B. Corresponding barrier heights for the other His64-side chain atoms range from 0.5 to 1.5 $\mathrm{kcal} / \mathrm{mol}$. Hence, on average state B is separated from state A by a barrier of $\approx 2 \mathrm{kcal} / \mathrm{mol}$ with state B lower in energy than state A.

The two states (A: green; B: red) are shown in Figure 4 together with the X-ray structure $1 \mathrm{HJT}^{43}$ (gold). State A ("His64 out") is associated with the short rebinding time scale (10 ps) whereas state B ("His64 in") corresponds to the slow (100 ps) component. The barrier height corresponds to an interconversion time $\mathrm{A} \leftrightarrow \mathrm{B}$ on the sub-nanosecond time scale which 

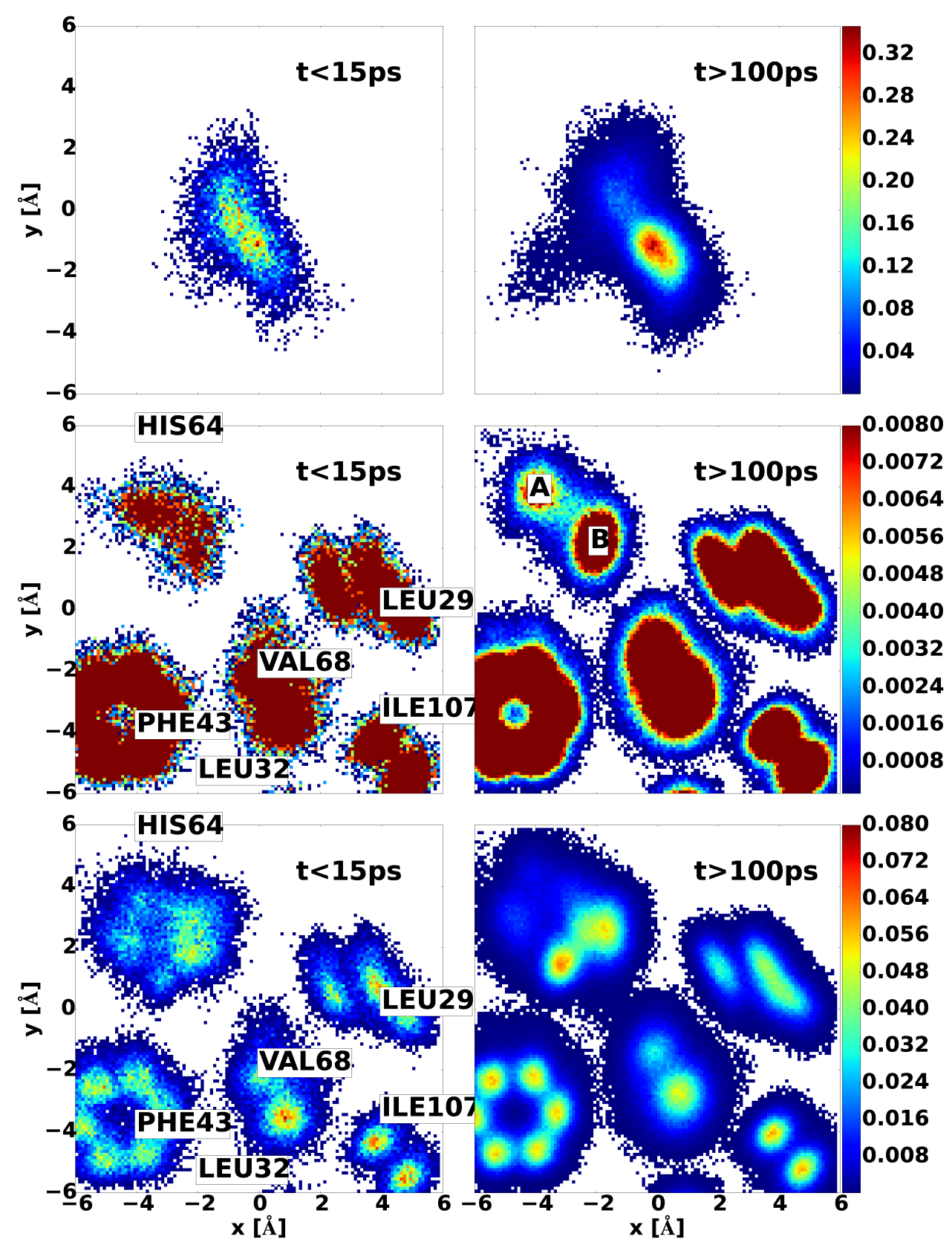

Figure 3: Top Panel: $(x, y)$-probability distribution function for the free NO ligand for rebinding on the $t<15 \mathrm{ps}$ (left) and $t>100 \mathrm{ps}$ (right) time scale. Middle Panel: $(x, y)$-probability distribution function for the NE2 atom of His64 on the two time scales. The two states for NE2 are labeled (A, B) and clearly distinguishable. Also shown are pdfs for all side chain atoms of Phe43, Val68, Leu29, and Ile107. For Phe43 the phenyl ring is always parallel to the heme-plane and all 6 carbon atoms are clearly distinguishable. Bottom Panel: as in the middle panel but for the entire side chain of His64 and with different maximum height of the pdfs. 
is supported by the explicit simulations and compares well with previous findings for CObound $\mathrm{Mb}$ with interconversion times of a few hundred picoseconds. ${ }^{6,44}$

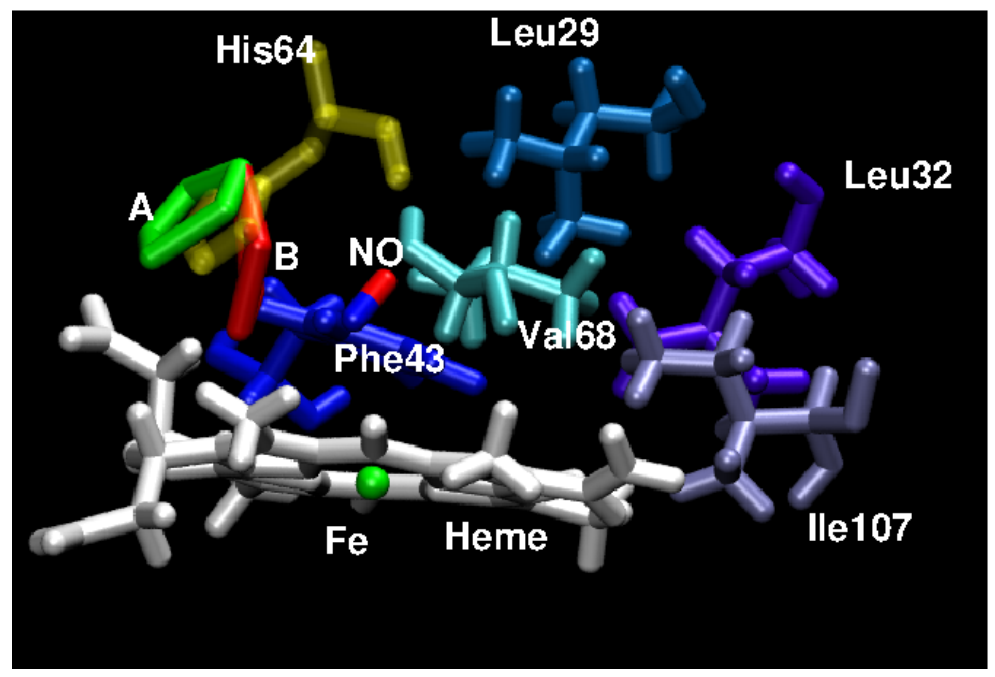

Figure 4: States A (green) and B (red) as found from the per-atom pdfs of the His64-side chain atoms. In gold the X-ray reference structure.

For direct contact with the XAS experiments, XANES spectra were computed (see SI) for randomly selected MD-sampled structures with NO-bound (40 structures), NO-unbound (NO within $3.5 \AA$ of the heme-Fe; 20 structures) and NO-unbound (NO within $5.0 \AA ; 10$ structures). For the bound state the computed spectrum $\left(I_{\mathrm{b}}(E)\right)$ agrees well up to $7.15 \mathrm{keV}$. For higher energies the absorption signal is correctly described but the computed intensity is too high (see Figure 5 top panel). The unbound structures yield $I_{\mathrm{ub}, \mathrm{s}}$ and $I_{\mathrm{ub}, \mathrm{l}}$ for the short and long $\mathrm{Fe}-\mathrm{NO}$ separations, respectively, and nearly superimpose (inset Figure 5) despite the different NO separations. Upon ligand photodissociation the peak at $7.12 \mathrm{keV}$ shifts to lower energy, the intensity between 7.12 and $7.15 \mathrm{keV}$ decreases and between 7.15 and $7.18 \mathrm{keV}$ increases compared to the spectrum for the bound state. All these features are consistent with previous experiments on $\mathrm{MbNO}$ and deoxy-Mb. ${ }^{5,14}$

The experimentally observed transient at $50 \mathrm{ps}$ is compared with the averaged absorbance differences $\Delta I_{\mathrm{ub}, \mathrm{s}}=I_{\mathrm{b}}-I_{\mathrm{ub}, \mathrm{s}}$ and $\Delta I_{\mathrm{ub}, \mathrm{l}}=I_{\mathrm{b}}-I_{\mathrm{ub}, \mathrm{l}}$ in Figure 5 . The two computed difference 

$\mathrm{keV}$ and an enhancement around $7.16 \mathrm{keV}$ for $\Delta I_{\mathrm{ub}, \mathrm{s}}$ compared to $\Delta I_{\mathrm{ub}, \mathrm{l}}$ - despite the two very different ligand positions. In both cases the Fe-oop position ranges from 0.3 to $0.15 \AA$ below the plane although for $\Delta I_{\mathrm{ub}, \mathrm{s}}$ positions closer to in-plane $(d=0)$ also occur. Compared to the experimental transient, $\Delta I_{\mathrm{ub}, \mathrm{s}}$ and $\Delta I_{\mathrm{ub}, \mathrm{l}}$ trace the major features but differ from it in the width of the $7.12 \mathrm{keV}$ peak and the behaviour between 7.13 and $7.15 \mathrm{keV}$. Figure S7 (see SI) suggests that presence or absence of photodissociated NO affects the XANES spectrum over the entire energy range from 7.1 to $7.15 \mathrm{keV}$ and not just around $7.15 \mathrm{keV}$ as previously assumed ${ }^{5}$ because the signal also depends on the motion of the Fe-atom relative to the heme-plane. Within the signal-to-noise of the experiment it can not be distinguished whether the ligand is close to the heme-Fe or further away from it. It should be recalled that experimentally, a mixture of NO-bound and NO-unbound structures is measured because the photolysis yield is not $100 \%$.

Reactive MD simulations using MS-ARMD yield nonexponential kinetics for ligand rebinding. The time scales (10 and 100 ps) confirm those from optical and infrared experiments. The influence of the Fe-oop coordinate on the rebinding reaction has been directly established. The two time scales are associated with two structurally different states of the His64 side chain - one "out" (state A) and one "in" (state B) - which control ligand access and rebinding dynamics. Although energetically feasible, the ${ }^{2} A \mathrm{Fe}-\mathrm{ON}$ metastable state is likely to be unobservable in wild type $\mathrm{Mb}$ because in this region of configurational space the repulsive ${ }^{4} A$ state is lower in energy and prevents stabilization. The present work supports a recently proposed, transient $\mathrm{Fe}-\mathrm{oop} / \mathrm{NO}-$ bound structure with lifetimes of up to $30 \mathrm{ps}$. The computed XAS spectra are compatible with experimentally measured ones but are unable to distinguish between structures with photodissociated NO "close to" or "far away" from the heme-Fe in the active site. The present work provides an atomistically refined picture and structural explanations and assignments for a range of experimental observations, all 


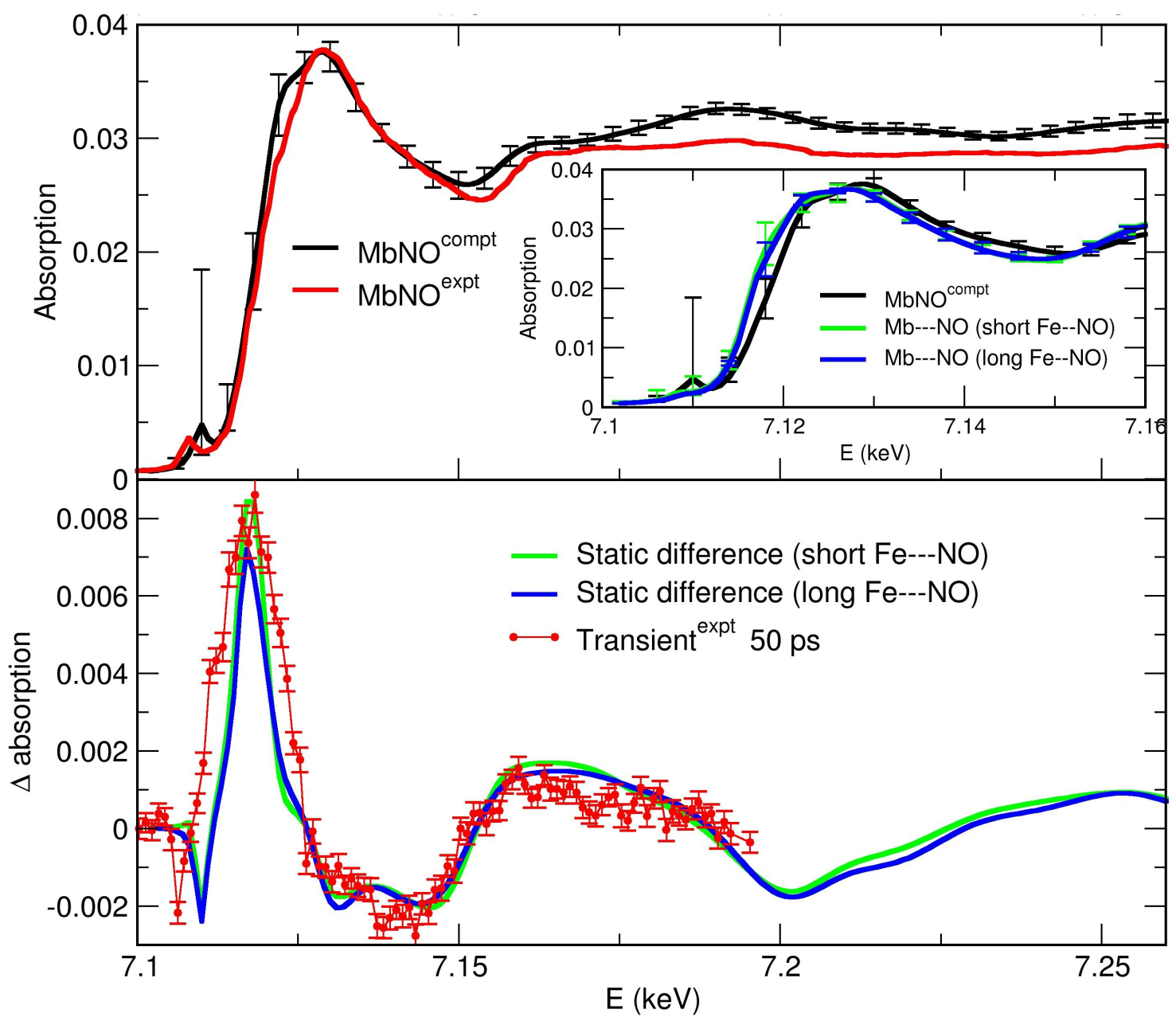

Figure 5: Top panel: Computed XANES spectra for MbNO (black) compared to experiment (red). Vertical bars indicate minimum and maximum absorption for all snapshots and are caused by conformational sampling. The inset compares computed spectra for MbNO and 2 sets of photodissociated systems (green and blue, see text). Bottom panel: Static difference spectra (green and blue) compared with the experimental transient at $50 \mathrm{ps}$ (red).

sensitive to the Fe-oop dynamics of $\mathrm{NO}$ after photodissociation in native $\mathrm{Mb}$.

\section{Acknowledgement}

The authors acknowledge fruitful discussions with Chris Milne and helpful comments from Peter Hamm and Majed Chergui. This work was supported by the Swiss National Science Foundation through grants 200021-117810, and the NCCR MUST. 


\section{References}

(1) Fischer, S.; Olsen, K. W.; Nam, K.; Karplus, M. Proc. Natl. Acad. Sci. 2011, 108, $5608-5613$.

(2) Menting, J. G. et al. Proc. Natl. Acad. Sci. 2014, 111, E3395-E3404.

(3) Schlichting, I.; Berendzen, J.; Phillips, G.; Sweet, R. Nature 1994, 371, 808-812.

(4) Schotte, F.; Lim, M.; Jackson, T. A.; Smirnov, A. V.; Soman, J.; Olson, J. S.; Phillips, G. N.; Wulff, M.; Anfinrud, P. A. Science (New York, N.Y.) 2003, 300, 1944.

(5) Silatani, M.; Lima, F. A.; Penfold, T. J.; Rittman, J.; Reinhard, M.; RittmannFrank, H.; Borca, C.; Grolimund, D.; Milne, C. J.; Chergui, M. Proc. Natl. Acad. Sci. 2015, 112, 12922 - 12927.

(6) Merchant, K. A.; Noid, W. G.; Thompson, D. E.; Akiyama, R.; Loring, R. F.; Fayer, M. D. J. Phys. Chem. B 2003, 107, 4-7.

(7) Bredenbeck, J.; Helbing, J.; Nienhaus, K.; Nienhaus, G. U.; Hamm, P. Proc. Natl. Acad. Sci. 2007, 104, 14243-14248.

(8) Pacher, P.; Beckman, J. S.; Liaudet, L. Physiol. Rev. 2007, 87, 315-424.

(9) Lundberg, J. O. et al. Nat. Chem. Biol. 2009, 5, 865-869.

(10) Traylor, T.; Sharma, V. Biochem. 1992, 31, 2847-2849.

(11) Cornelius, P.; Hochstrasser, R.; Steele, A. J. Mol. Biol. 1983, 163, 119-128.

(12) Petrich, J. W.; Lambry, J. C.; Kuczera, K.; Karplus, M.; Poyart, C.; Martin, J. L. Biochem. 1991, 30, 3975.

(13) Ionascu, D.; Gruia, F.; Ye, X.; Yu, A.; Rosca, F.; Demidov, C. B. A.; Olson, J. S.; Champion, P. M. J. Am. Chem. Soc. 2005, 127, 16921-16934. 
(14) Kruglik, S. G.; Yoo, B.-K.; Franzen, S.; Vos, M. H.; Martin, J.-L.; Negrerie, M. Proc. Natl. Acad. Sci. 2010, 10\%, 13678.

(15) Kim, J.; Park, J.; Lee, T.; Lim, M. J. Phys. Chem. B 2012, 116, 13663-13671.

(16) Yoo, B.-K.; Kruglik, S. G.; Lamarre, I.; Martin, J.-L.; Negrerie, M. J. Phys. Chem. B 2012, 116, 4106-4114.

(17) Kim, S.; Jin, G.; Lim, M. J. Phys. Chem. B 2004, 108, 20366.

(18) Nutt, D. R.; Meuwly, M. Biophys. J. 2006, 90, 1191.

(19) Danielsson, J.; Meuwly, M. J. Chem. Theo. Comp. 2008, 4, 1083.

(20) Nienhaus, K.; Palladino, P.; Nienhaus, G. U. Biochem. 2008, 47, 935.

(21) Xu, N.; Yi, J.; Richter-Addo, G. B. Inorg. Chem. 2010, 49, 6253-6266.

(22) Nutt, D. R.; Karplus, M.; Meuwly, M. J. Phys. Chem. B 2005, 109, 21118.

(23) Nutt, D. R.; Meuwly, M. Proc. Natl. Acad. Sci. 2004, 101, 5998.

(24) Lee, M. W.; Carr, J. K.; Göllner, M.; Hamm, P.; Meuwly, M. J. Chem. Phys. 2013, $139,054506$.

(25) Soloviov, M.; Meuwly, M. J. Chem. Phys. 2015, 143.

(26) Aronszajn, N. Trans. Amer. Math. Soc. 1950, 68, 337.

(27) Hollebeek, T.; Ho, T. S.; Rabitz, H. Ann. Rev. Phys. Chem. 1999, 50, 537.

(28) Ho, T. S.; Rabitz, H. J. Chem. Phys. 1996, 104, 2584.

(29) Meuwly, M.; Hutson, J. M. J. Chem. Phys. 1999, 110, 8338.

(30) Meuwly, M.; Becker, O. M.; Stote, R.; Karplus, M. Biophys. Chem. 2002, 98, 183-207. 
(31) Lim, M.; Jackson, T. A.; Anfinrud, P. A. Proc. Natl. Acad. Sci. 1993, 90, 5801.

(32) Nutt, D. R.; Meuwly, M. Biophys. J. 2006, 90, 1191-1201.

(33) Bossa, C.; Anselmi, M.; Roccatano, D.; Amadei, A.; Vallone, B.; Brunori, M.; Di Nola, A. Biophys. J. 2004, 86, 3855.

(34) Plattner, N.; Meuwly, M. Biophys. J. 2012, 102, 333.

(35) Kholodenko, Y.; Gooding, E.; Dou, Y.; Ikeda-Saito, M.; Hochstrasser, R. Biochem. 1999, 38, 5918-5924.

(36) Ye, X.; Demidov, A.; Champion, P. J. Am. Chem. Soc. 2002, 124, 5914-5924.

(37) Kim, S.; Jin, G.; Lim, M. J. Phys. Chem. B 2004, 108, 20366-20375.

(38) Banushkina, P.; Meuwly, M. J. Chem. Phys. 2007, 127.

(39) Carducci, M.; Pressprich, M.; Coppens, P. J. Am. Chem. Soc. 1997, 119, 2669-2678.

(40) Cheng, L.; Novozhilova, I.; Kim, C.; Kovalevsky, A.; Bagley, K. A.; Coppens, P.; Richter-Addo, G. B. J. Am. Chem. Soc. 2000, 122, 7142.

(41) Hunt, N. T.; Greetham, G. M.; Towrie, M.; Parker, A. W.; Tucker, N. P. Biochem. J. 2011, 433, 459-468.

(42) Kepp, K. P. Bioch. Bioph. Acta 2015, 1854, 1239-1248.

(43) Brucker, E. A.; Olson, J. S.; Ikeda-Saito, M.; Phillips, G. N. Prot. Struct. Funct. Genet. 1998, 30, 352 .

(44) Ma, J.; Huo, S.; Straub, J. J. Am. Chem. Soc. 1997, 119, 2541-2551. 